\title{
CONSTRAINED VAPOR BUBBLE
}

\section{J. Huang, M. Karthikeyan, J. Plawsky, P. C. Wayner, Jr., The Isermann Department of Chemical Engineering, Rensselaer Polytechnic Institute, Troy NY12180, USA, wayner@rpi.edu}

\begin{abstract}
The nonisothermal Constrained Vapor Bubble, CVB, is being studied to enhance the understanding of passive systems controlled by interfacial phenomena. The study is multi-faceted: 1) it is a basic scientific study in interfacial phenomena, fluid physics and thermodynamics; 2) it is a basic study in thermal transport; and 3 ) it is a study of a heat exchanger. The research is synergistic in that CVB research requires a microgravity environment and the space program needs thermal control systems like the CVB.

Ground based studies are being done as a precursor to flight experiment. The results demonstrate that experimental techniques for the direct measurement of the fundamental operating parameters (temperature, pressure, and interfacial curvature ficlds) have been developed. Fluid flow and change-of-phase heat transfer are a function of the temperature field and the vapor bubble shape, which can be measured using an Image Analyzing Interferometer.
\end{abstract}

\section{INTRODUCTION}

The CVB, which is presented in Fig. 1 for a microgravity environment, has various thin film regions that are of both basic and applied interest. Generically, a CVB is formed by underfilling an evacuated enclosure with a liquid. Classification depends on shape and Bond number. The specific CVB discussed herein was formed in a fused silica cell with inside dimensions of $3 \times 3 \times 40 \mathrm{~mm}$ and, therefore, can be viewed as a large version of a micro heat pipe. Since the dimensions are relatively large for a passive system, most of the liquid flow occurs under a small capillary pressure difference. Therefore, we can classify the discussed system as a low capillary pressure system. The studies discussed herein were done in a $1 g$ environment (Bond Number $=3.6$ ) to obtain experience to design a microgravity experiment for a future NASA flight where low capillary pressure systems should prove more useful. The flight experiment is tentatively scheduled for the year 2000 . The SCR was passed on September 16, 1997. The RDR is tentatively scheduled for October, 1998.

Since the solid walls of the container constrain the shape of the vapor bubble and liquid/vapor interface, the intermolecular force ficlds in the thin film regions are different from that in a bulk liquid. In addition to surface tension stresses (c.g., Marangoni stresses), both

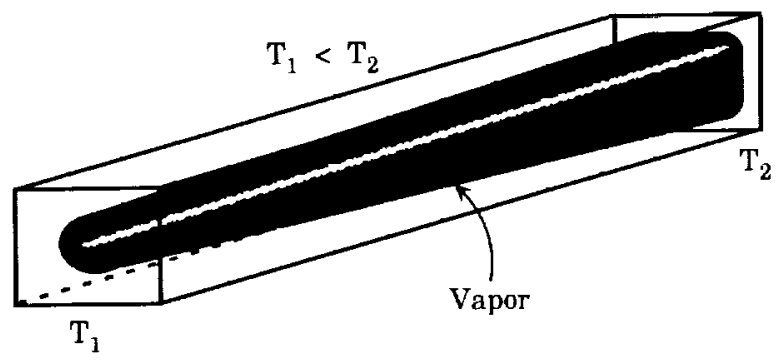

Figure 1: Vapor bubble constrained in glass cell.

disjoining pressure (due to van der Waals forces) and capillary pressurc gradients are important (Potash and Wayner, 1972). For a completely wetting system with insufficient liquid to fill the cell, the liquid will coat all the walls of the container. For a particular liquid/solid system, the film thickness profile is a function of the fill ratio and temperature and concentration profiles.

Under equilibrium conditions, the CVB system has been used to study interfacial thermodynamics and characterize wetting (DasGupta et al., 1995). For the nonequilibrium case presented in Fig. 1, there is an external heat input at End (2), where evaporation occurs, and heat removal near End (1), where condensation occurs. The return condensate flows from End (1) to End (2) as a result of stress gradients due to interfacial frec energy (e.g., capillary and disjoining pressure gradients). For a very small cross-sectional area with a mean meniscus curvature comparable to the reciprocal of the hydraulic radius of the flow channel, and Bond number $\approx 4 \times 10^{-3}$, the system has been called a micro heat pipe, which has been extensively studied (Cotter, 1984, Peterson, 1992, and Faghri, 1995). Under some conditions, the processes are like those in other heat transfer systems, c.g., the microlayer in boiling. Thereforc, the experimental and theoretical results obtained studying the CVB are of both generic and specific use.

The CVB study is multi-faceted: 1) it is a basic scientific study in interfacial phenomena, fluid physics and thermodynamics; 2) it is a basic study in thermal transport; and 3) it is a study of a heat exchanger. The research is synergistic in that CVB research requires a microgravity environment and the space program needs thermal control systems like the CVB. The objective of this paper is to present some of our ground based results. 


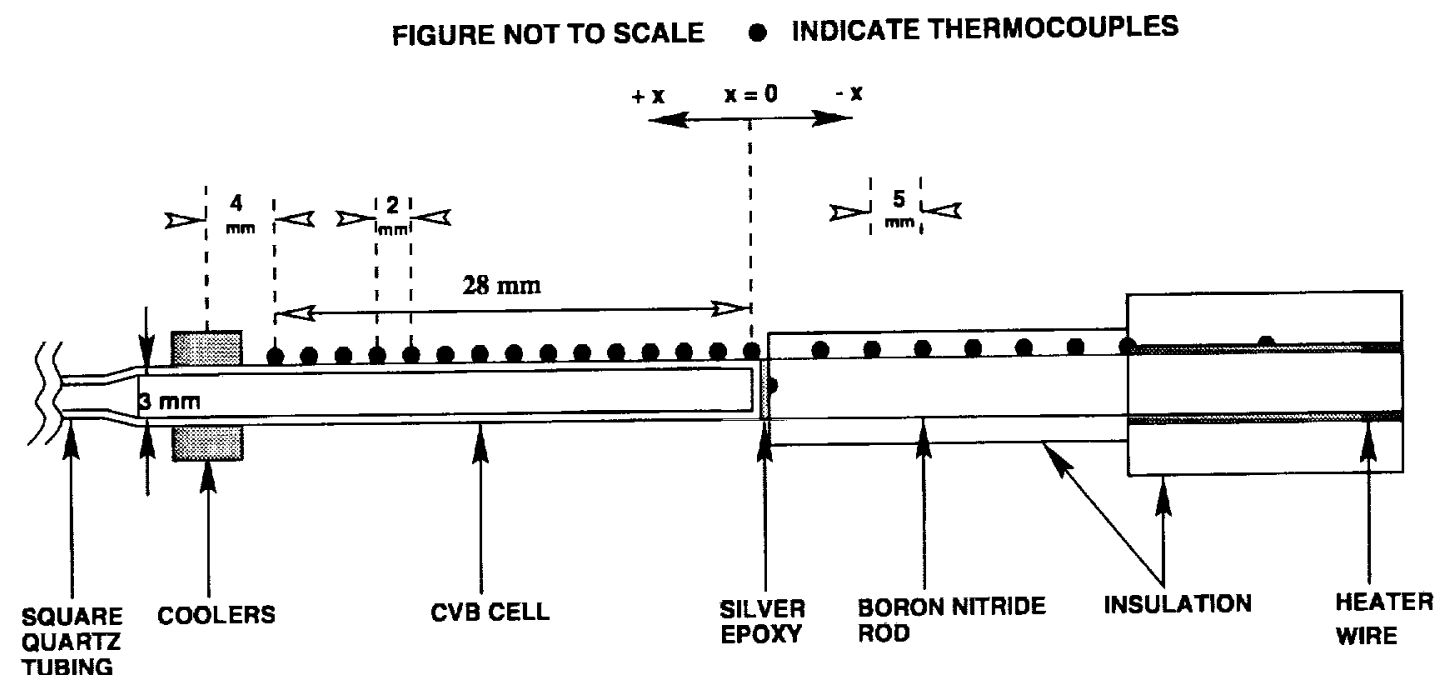

Figure 2: Experimental sctup.

These results demonstrate that experimental techniques for the direct measurement of the fundamental operating parameters (temperature, pressure, and interfacial curvature fields) of the system have been developed. Fluid flow and change-of-phase heat transfer are a function of the lemperature field and the vapor bubble shape, which can be measured using an Image Analyzing Interferometcr. The extended Young-Laplace Equation gives the pressure field as a function of the shape.

\section{EXPERIMENTAL METHOD}

A pentane/quartz CVB was used to study transport processes under non-equilibrium conditions (Karthikcyan et al., 1997 and Huang et al., 1997). In Fig. 2, the experimental setup consists mainly of the fused silica CVB cell $(3 \times 3 \times 40 \mathrm{~mm}$ inside dimensions with walls $1.25 \mathrm{~mm}$ thick), thermocouples to measure the temperature on the outside surface, $T$, a resistance heater, and thermoelectric coolers. Pyrex connecting tubes and a pressure transducer to measure the vapor pressure are not shown. Additional details of the experimental equipment are presented by Karthikeyan (1997). The experimental use of glass allows the profile and, therefore, the pressure gradient causing fluid flow to be optically measured using a microscope. A portion of the optical system to record the naturally occurring fringes in the top corner of the cell to obtain $\delta_{0}$ and $r$ is presented in Fig. 3. Details of this film thickness measurement technique, Image Analyzing Interferometry (IAI), are claborated elsewhere (DasGupta et al., 1995).

Experimentally, we have found that, in the low cap- illary pressure systems of interest, dramatically different phenomena occur on the top and bottom surfaces because of the gravitational force field. Therefore, the process on one surface interferes with the study of the process on the other process. For example, at times, boiling occurs on one surface while not on the other. A significant change in the system size to obtain a lower Bond Number by increasing the capillary pressure would also produce a significant change in the phenomena being studied. Wc are interested in the lower capillary pressure system with lower viscous effects due to their relatively large size.

In Fig. 2, a CVB cell is connected to a boron nitride rod. The measured boron nitride rod temperatures were fitted to the classical fin equation. The heat going out from the end of the boron nitride $\operatorname{rod}(x=-1 \mathrm{~mm}), Q_{o u t}$, was calculated from the gradient of the temperature profile. The heat input to the CVB cell at $x=0 \mathrm{~mm}$, $Q$, was obtained by deducting the calculated convective heat loss from the first $\mathrm{mm}$ of the CVB cell from $Q_{\text {out }}$. The overall uncertainties in Q, calculated using the classical propagation equation, was about $18 \%$ (see Karthikeyan, 1998).

\section{EXPERIMENTAL RESULTS AND DIS- CUSSIONS}

Fig. 4 shows a plot of the temperature difference, $\theta$, between the outside surface of the CVB cell and the room, $v s$. the axial distance, $x$, for both the cell operating as a CVB, and as a dry evacuated cell. $x=0$ represents the beginning of the cavity of the cell and 
CONSTRAINED VAPOR BUBBLE: J. Huang et al.

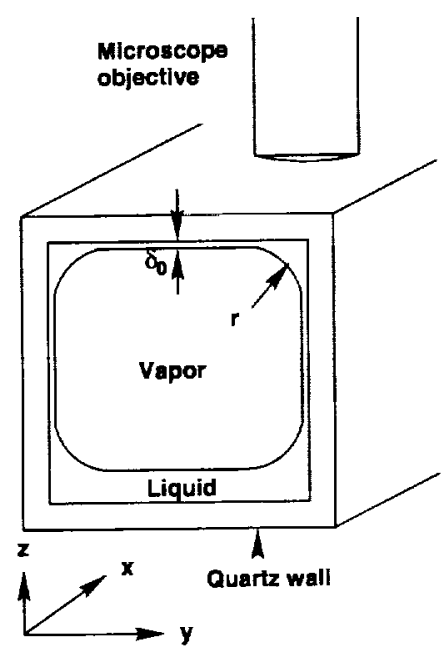

(a)

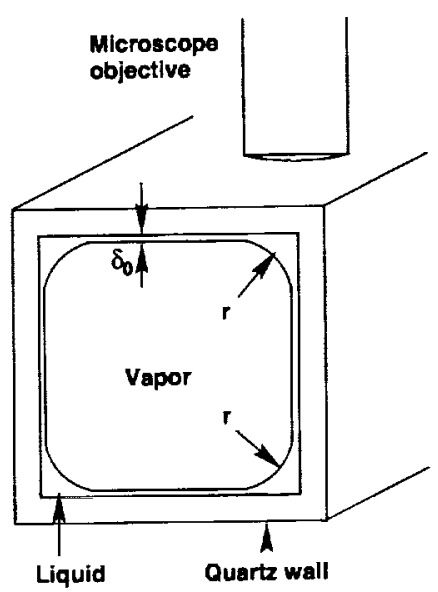

(b)

Figure 3: Location of the microscope objective with respect to the CVB cell: (a) asymmetric film in $1 \mathrm{~g}$ environment: (b) filn with Bond Number $=0$.

the two dashed lines represent the $1 \mathrm{~mm}$ thick glass wall at the heater end of the cell. The electrical power inputs to the heater (13.4 W) and coolers were the same in both cases. However, we see a dramatic difference between the temperature profiles of the CVB and the dry run. While the temperature profile for the dry cell resembles that of a fin, the CVB temperature profile has three distinct regions (shown in Fig. 5). The heat flow rate through the CVB is almost three times that of the heat flow rate through the dry cell ( $1.49 \mathrm{~W}$ for the CVB against $0.5 \mathrm{~W}$ for the dry cell). We define the thermal conductance, $C$, as the ratio of the CVB heat input to the total temperature drop across the length of the cell,

$$
C=Q / \Delta T
$$

At $Q=0.5 \mathrm{~W}$, we found that the conductance for the dry cell was $0.0036 \mathrm{~W} / \mathrm{K}$ versus $0.01 \mathrm{~W} / \mathrm{K}$ for the $\mathrm{CVB}$, a three-fold increase in the conductance for the CVB.

A comparison of the measured radius of curvature, $r$, profile in the upper corner for a non-isothermal CVB experiment $(\mathrm{Q}=0.81 \mathrm{~W})$ to that for an isothermal $\mathrm{CVB}$ cell, is shown in Fig. 6 . In both cases, the bubble length was $26 \mathrm{~mm}$ and the angle of inclination was $-4^{\circ}$. The difference in the two profiles is obvious; while the radius of curvature for the isothermal case changes relatively little, the radius of curvature for the non-isothermal case increases from about $0.09 \mathrm{~mm}$ to more than five times as much. The increased capillary pressure gradient is needed to recirculate the working fluid. On the other hand, the change in the radius of

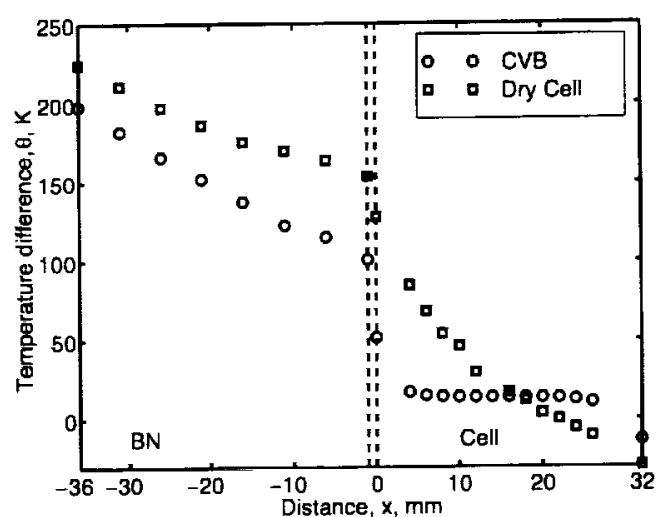

Figure 4: Temperature difference profiles of the CVB and the corresponding dry cell $\left(\theta=T-T_{\infty}\right)$.

curvature in the isothermal case was caused solely by the gravitational force. Due to gravitational effects, the radius of curvature profile in the bottom of the cell is different. Fig. 7 shows both the experimental radii of curvature and those predicted by the model (Karthikeyan et al., 1998). There is good agreement between these experimental and predicted values. Obviously, the gradient of the radius of curvature profile in higher for the higher heat flow rate experiment.

In order to characterize the variation in the effectiveness of the CVB from region to region, we define 


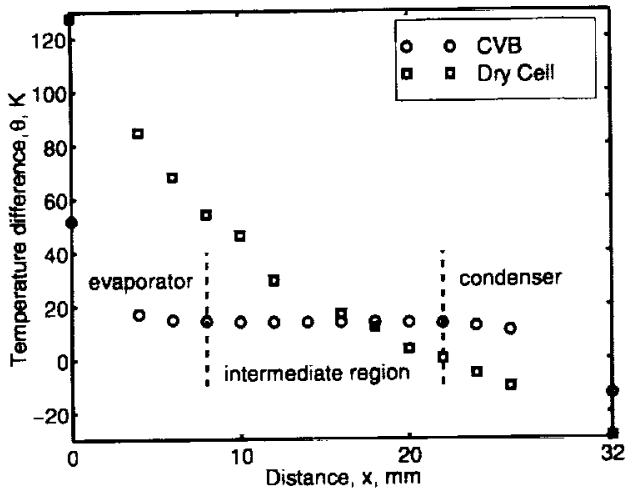

Figure 5: Temperature difference profiles in the quartz cell for the experiments shown in Fig. $4\left(\theta=T-T_{\infty}\right)$.

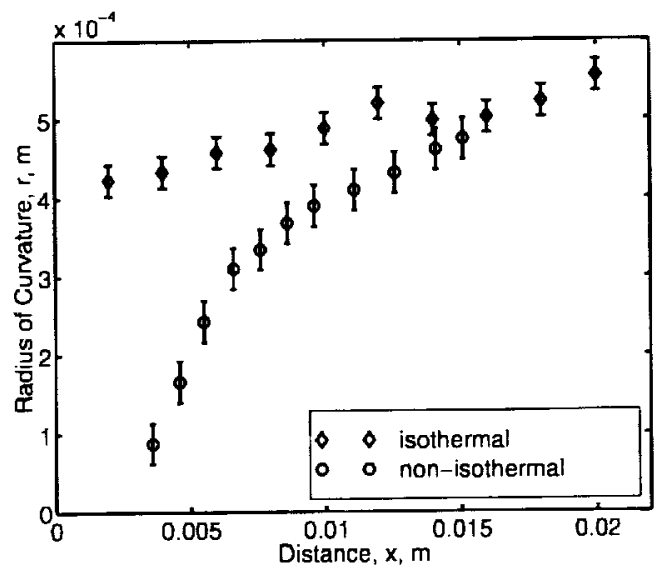

Figure 6: Corner radius of curvature profiles obtained using IAI.

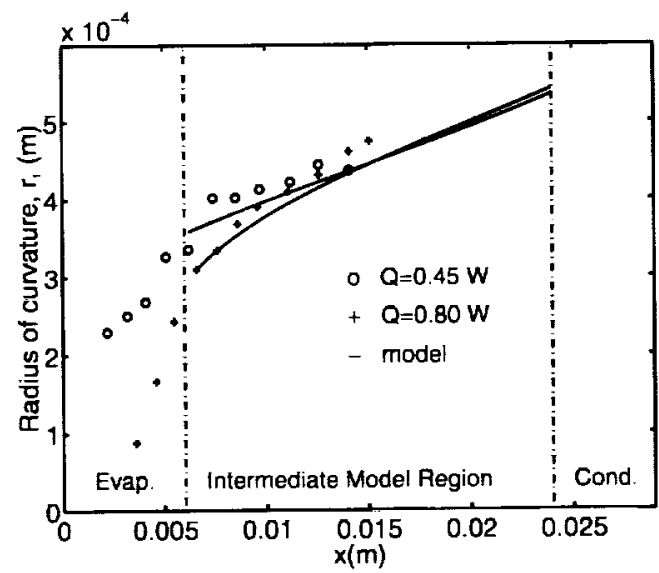

Figure 7 : Radii of curvature profiles for bubble length $=26 \mathrm{~mm}$ and the angle of inclination $=-4^{\circ}$.

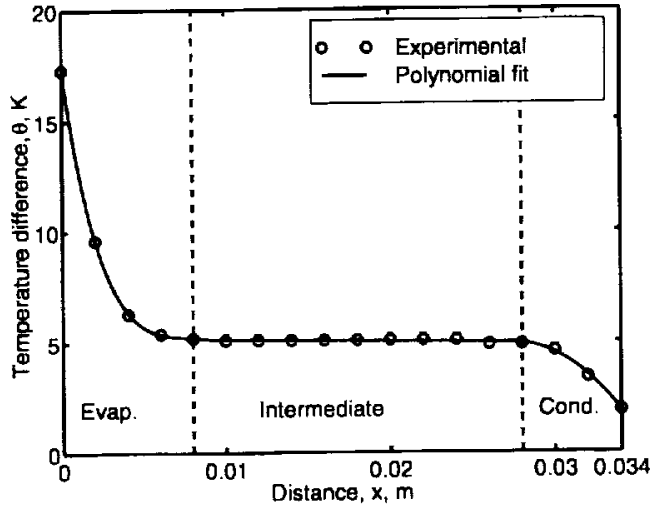

Figure 8: CVB temperature difference profile and 3region polynomial fit $\left(0=T-T_{\infty}\right)$.

the local effective thermal conductivity, $k_{e f f}(x)$ as,

$$
k_{c f f}(x)=-\frac{q(x)}{d \theta / d x}
$$

where

$$
q(x)=\frac{Q-\int_{0}^{x} L_{q} h_{c e l l} \theta d x}{A}
$$

where $L_{q}$ is the outside perimeter of the cell, $A$ the crosssectional area of the cell, and the heat transfer coefficient between the cell and the environment, $h_{c c l l}$, which was obtained from an equivalent dry cell experiment. The plot of the temperature profile of one CVB experiment $(\mathrm{Q}=0.316 \mathrm{~W})$ along with the polynomial fit is shown in Fig. 8, while the plot of the local effective thermal conductivity, $k_{e f f}$, as a function of position, $x$, is shown in Fig. 9. Even including the area of the glass which has a very low thermal conductivity, the effective thermal conductivity (solid line in Fig. 9) in the intermediate region well exceeds the thermal conductivity of copper depicted by the dash-dot line.

Figs. 10 and 11 show the temperature profiles for CVB experiments at various heat loads with bubble length, $l_{b}, 26 \mathrm{~mm}$ and $>28 \mathrm{~mm}$, respectively. The fixed volume of the system could be experimentally set. When the bubble length is greater than $28 \mathrm{~mm}$, the bubble extends all the way to the coolers. The reduced thermal resistance in the condenser region allows a higher heat flow rate to the CVB cell. Fig. 12 shows a plot of the thermal conductance, $C$ vs. the heat load, $Q$, for the experiments shown in Figs. 10 and 11 . Since the conductance increases with an increase in the heat load, the CVB has not reached its capillary limit.

Fig. 13 shows an excellent correlation between the CVB operating temperature and the saturation temper- 
CONSTRAINED VAPOR BUBBLE: J. Huang et al.

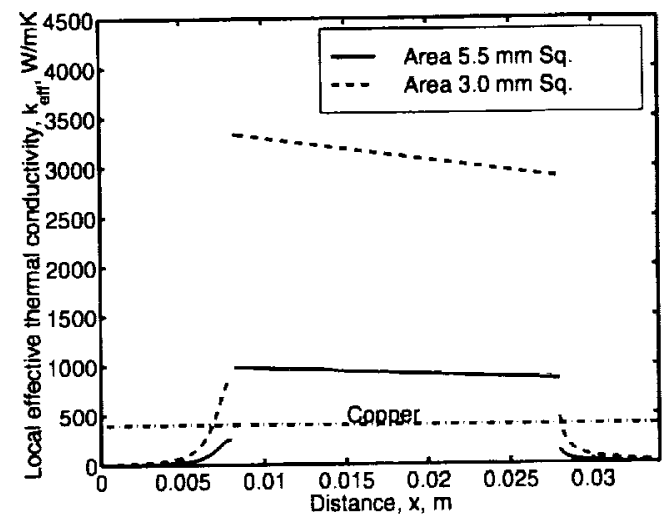

Figure 9: Local effective thermal conductivity vs. $x$ for the CVB.

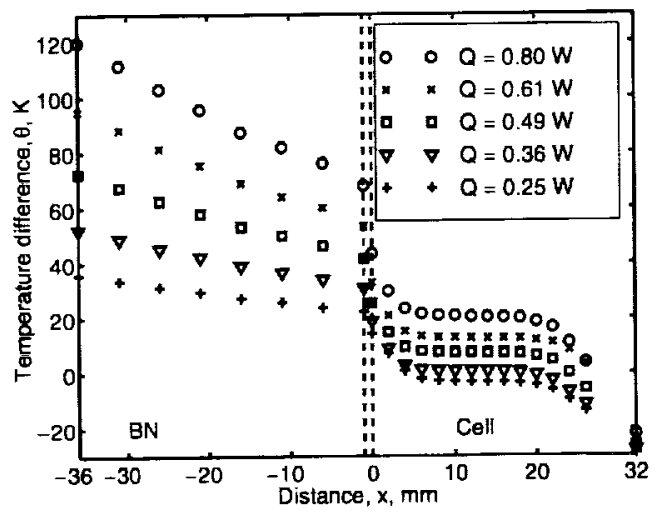

Figure 10: Temperature difference profiles of CVB for various $\mathrm{Q}, l_{b}=26 \mathrm{~mm}\left(\theta=1-T_{\infty}\right)$.

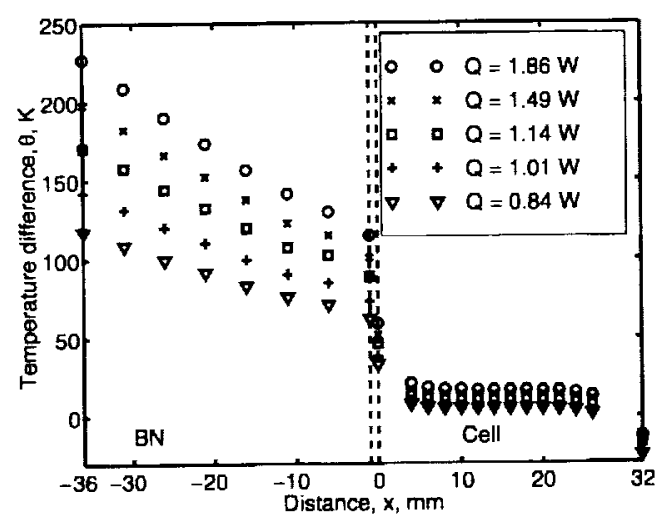

Figure 11: Temperature difference profiles for $l_{b}>$ $28 \mathrm{~mm}$ (constant but not exactly known), $\left(\theta=T-T_{\infty}\right)$.

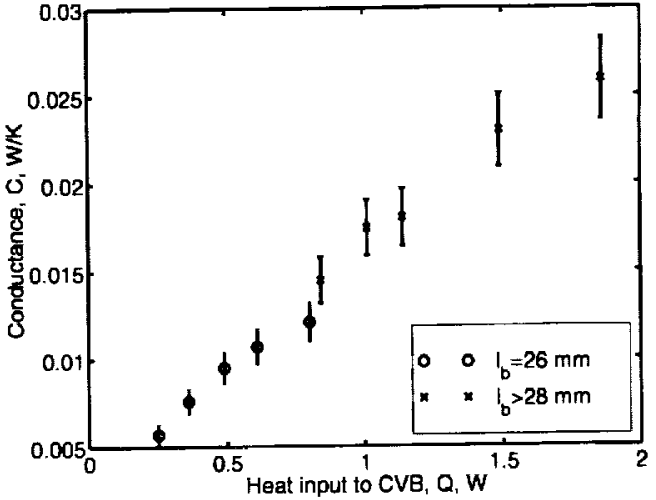

Figure 12: Thermal conductance as a function of the heat input to the CVB.

ature based on the measured pentane vapor pressures. However, it is also seen that the saturation temperature, $T_{s a t}$, is always a little higher than the measured outside temperature in the intermediate region, $T_{i n t}$, where radial heat conduction through the glass wall is very small. This temperature difference, which never exceeds 2.5 ${ }^{\circ} \mathrm{C}$, may be due to non-condensables or the heat loss from the intermediate region. Kelvin effects at these capillary pressures are very small.

Fig. 14 shows the CVB temperature profiles for experiments conducted at various angles of inclination, under constant heater and cooler power inputs. The bubble length was also a constant at $26 \mathrm{~mm}$. The negative sign of the angle means that the evaporator is at a higher level than that of the condenser, and capillary forces are hindered by the hydrostatic head in the flow of the condensate back to the evaporator. As we can see from Fig. 14, both length and temperature of the intermediate region decrease with an increase in the angle of inclination from $0^{\circ}$ to $-25^{\circ}$. In fact, for the experiment at $-25^{\circ}$, the region of small temperature gradient (intermediate region) is almost non-existent. Additionally, it is seen that the heat input to the CVB decreases with an increase in the angle of inclination. This is because of the reduction in the liquid flow rate from the condenser to the evaporator due to the opposition of the hydrostatic head to liquid flow. Since the heat removal rate through the coolers remains the same, a reduction in the heat input with the increase in the angle of inclination causes a reduction in the operating pressure; consequently, the operating temperature of the CVB decreases. As expected, the thermal conductance of the CVB decreases with an increase in the angle of inclination. This trend is plotted in Fig. 15. 


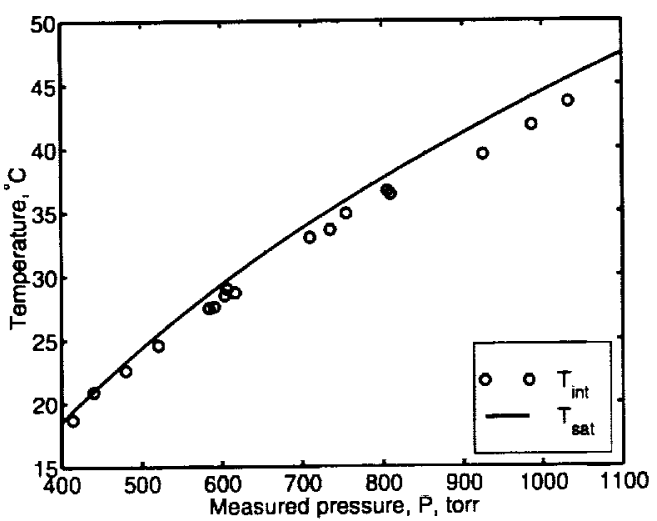

Figure 13: Variation of the CVB operating temperature with operating pressure.

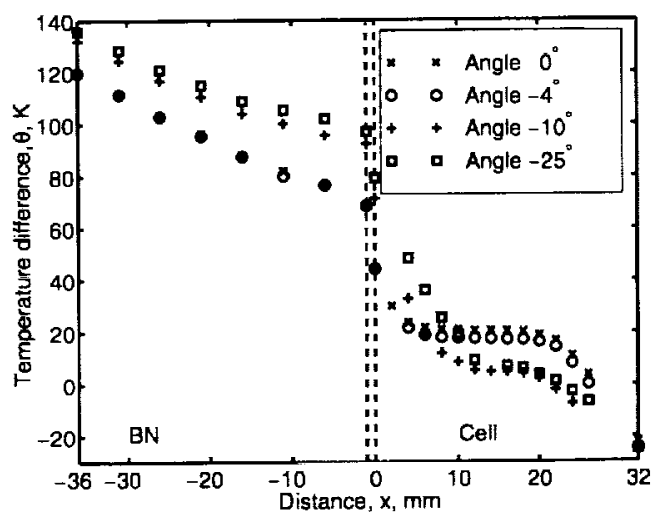

Figure $14: \theta=T-T_{\infty}$ profiles $(Q=0.80,0.71,0.48,0.33$ $W$, for angles $=0^{\circ},-t^{\circ},-10^{\circ},-25^{\circ}$, respectively).

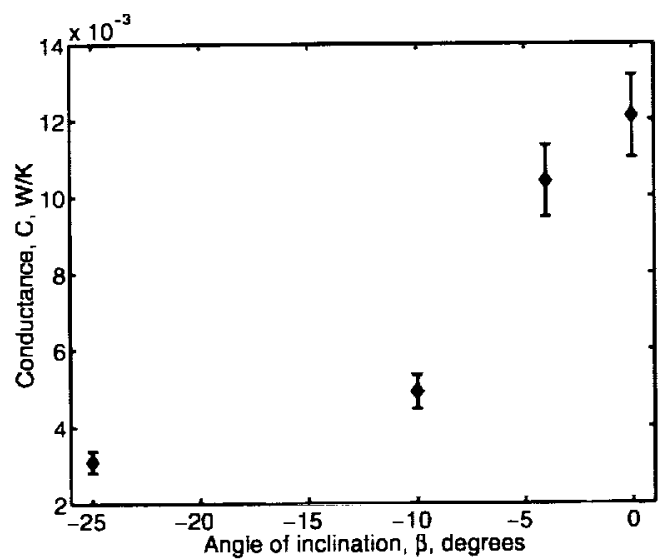

Figure 15: Variation of thermal conductance with the angle of inclination of the CVB.

\section{CONCLUSIONS}

1) Experimental techniques for direct measurement of the fundamental operating parameters (temperature, pressure and interfacial curvature fields) in the Nonisothermal Constrained Vapor Bubble (CVB) were demonstrated.

2) The CVB has an overall thermal conductivity that is extremely high in the intermediate region of the cell where heat losses to the surroundings are minimized.

3) For the heat flow rates studied, the thermal conductance of the CVB increases with an increase in the heat input.

4) The performance of the CVB is a strong function of its orientation with respect to gravity.

\section{ACKNOWLEDGMENT}

This material is based on work supported by the National Aeronautics and Space Administration under grant \# NAG3-1834. Any opinions, findings, and conclusions or recommendations expressed in this publication are those of the authors and do not necessarily reflect the view of NASA.

\section{REFERENCES}

Cotter, T. P., 1984, Principles and Prospects of Micro Heat Pipes, Proc. 5th Int. Heat Pipe Conf., Tsukuba, Japan, pp. 328-335.

DasGupta, S., Plawsky, J. L., and Wayner, P. C., Jr., 1995, Interfacial Force Field Characterization in a Constrained Vapor Bubble Thermosyphon, AIChE Journal, 41(9), pp. 2140-2149.

Faghri, A., 1995, Heat Pipe Science and Technology, First Edition, Taylor and Francis, Washington, DC.

Huang, J., Karthikeyan, M., Plawsky, J. L., and Wayner, P. C., Jr., 1997, Two-Dimensional Analysis of the Evaporator of a Constrained Vapor Bubble, Proc. 10th Intl. Heat Pipe Conf.(F-5), Stuttgart, Germany.

Karthikeyan, M., 1997, Experimental and Analytical Investigation of the Non-Isothermal Constrained Vapor Bubble, Ph.D. Thesis, Department of Chemical Engineering, Rensselaer Polytechnic Institute, NY

Karthikeyan, M., Huang, J., Plawsky, J. L., and Wayner, P. C., Jr., 1998, Experimental Study and Modeling of the Intermediate Section of the Non-isothermal Constrained Vapor Bubble, J. Heat Transfer Vol. 120, pp. 166-173.

Peterson, G. P., 1992, Overview of Micro Heat Pipe Rescarch and Development, Appl. Mech. Rev, 45(5), pp. 175-189.

Potash, M. Jr. and Wayner, P. C. Jr., 1972, Evaporation from a Two-Dimensional Extended Meniscus, Int. J. Heat Mass Transfer, Vol. 15, pp. 1851-1863. 